\title{
DESIGN SYNTHESIS AND EXPERIMENTAL VALIDATION OF MICROFLUIDIC CONCENTRATION GRADIENT GENERATORS
}

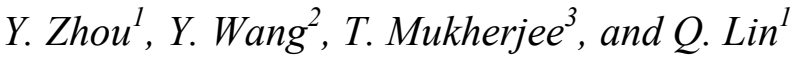 \\ ${ }^{1}$ Columbia University, New York, New York, USA \\ ${ }^{2}$ CFD Research Corporation, Huntsville, Alabama, USA \\ ${ }^{3}$ Carnegie Mellon University, Pittsburgh, Pennsylvania, USA
}

\begin{abstract}
This paper presents a synthesis method and its experimental validation for microfluidic concentration gradient generators that use multi-stream laminar flow. The method is based on an iterative framework in which designs are evaluated with an analytical convection-diffusion model. Comparing to the expensive trial-and-error experimentation or the time consuming finite element simulation, this design scheme enables the efficient design of concentration gradient generators capable of generating complex concentration profiles. We describe the synthesis method, and validate it by experiments with devices that are designed based on the method. The concentration profiles obtained from the experiments agree well with the prescribed ones.
\end{abstract}

\section{INTRODUCTION}

Concentration gradients of chemical reagents play important roles in cell biology studies[1-3]. Conventionally, the Boyden chambers, pipettes and gels are used to create concentration gradients of chemicals. However, these methods usually fail to maintain spatial and temporal stability of the gradient due to uncontrolled sample injection and transport, and hence compromise the accuracy of the experiments. The advent of microfluidic device enables a significantly greater control over the chemical environment. These devices allow accurate and reproducible manipulation of samples, and thus provide a powerful tool to create concentration gradients of chemicals for cell studies.

Microfluidic concentration generating devices are commonly based on laminar diffusion. Generally, these devices can be classified into to two categories: complete mixing- and partial-mixing based. Complete mixing devices usually have a configuration of hierarchical micro-channel networks [4-6]. In each hierarchy, sample solutions and buffer are recombined, mixed and splitted. Sufficient sample mixing before each splitting is required to produce a uniform concentration across the channel width. For this reason, such gradient generators often use bulky micro-channel networks that are prone to leakage and clogging.

In comparison, concentration gradient generators fully relying on diffusion-based partial-mixing are simpler and more reliable. A Y-shape diffusion diluter [7] and a crossmixing microfluidic device [1] were reported, both utilizing partial mixing of samples to create simple channelwidthwise concentration profiles. In these devices, samples and buffers simply mix as they converge and flow down the channel. At all locations along the channel, stable transverse profiles are achieved as a result of the balance between convection and diffusion. To enable the efficient design of this type of devices for more complex concentration profiles, an analytical convection-diffusion model was proposed [8]. Although the calculation results from the analytical model were compared to numerical simulations, no systematic experimental data were available to verify the model.

In this paper, we first formulate a synthesis scheme which incorporates the above-mentioned analytical model to generate a design for a user-specified complex profile, i.e. given a desired concentration profile, design for the appropriate device geometries and experimental parameters. Then, based on the proposed design scheme, we design and fabricate microchips, and carry out experiments to validate the analytical model as well as our synthesis method.

\section{DESIGN SYNTHESIS}

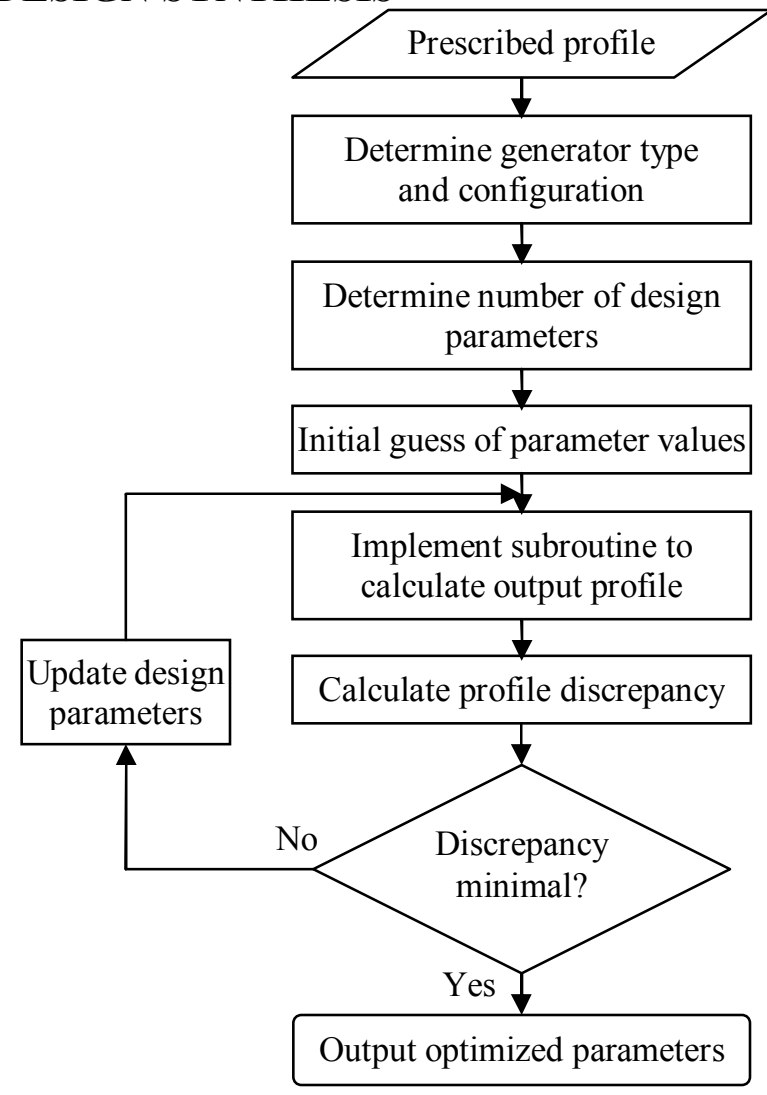

Fig. 1: Concentration gradient generator synthesis process.

The synthesis method is an iterative optimization process which is represented in a flowchart (Fig. 1). A complex concentration profile is generally decomposed into simple "building block" profiles, i.e. linear and bell-shape profiles. Given a prescribed profile, designers first need to determine the types of constitutive elements for the device (e.g. Y-mixer for linear profile or cross-mixer for bell-shape 
profile), device configuration (e.g. double cross or triple Y), and design parameters (e.g. channel dimensions, concentration or flow rate at each inlet). Then, with the initial guess values of the parameters, the modeling subroutine calculates the output concentration profile at a specified location as well as its discrepancy from the desired profile, using the analytical model of Ref. [8]. Based on the discrepancy, the parameters are then adjusted and input into the modeling subroutine again for a second iteration of calculation. This step progresses until the optimal parameter values is achieved which yield minimal discrepancy.

We create three design cases, each representing a distinct type of concentration profile. First, for the main purpose of verifying the analytical model, we use the elementary profiles, i.e. linear profiles (denoted as L-1 and L-2, e.g. Fig. 5) and bell-shape profiles (denoted as BS-1, BS-2 and BS-3, e.g. Fig. 6) in the first two case studies. To validate the synthesis method, the third and more complex profiles we select is piecewise linear (denoted as PL-1 and PL-2, e.g. Fig. 7), which combine three consecutive single linear profiles. The mathematical expressions of linear, bell-shape, and piecewise linear profiles are represented as

$$
\begin{gathered}
c=a+b x \\
c=\left\{\begin{array}{lc}
\operatorname{Erf}\left(B_{1}\left(x-B_{2}\right)\right)-\operatorname{Erf}\left(B_{1}\left(x-\left(1-B_{2}\right)\right)\right) \\
2 \\
a_{1}+b_{1} x & 0 \leq x<1 / 3 \\
a_{2}+b_{2} x & 1 / 3 \leq x<2 / 3 \\
a_{3}+b_{3} x & 2 / 3 \leq x \leq 1
\end{array}\right.
\end{gathered}
$$

where in all three equations, $x$ is the normalized lateral position of a point with reference to the left and right channel walls, and $c$ is the normalized concentration at point $x$.with reference to a standard concentration. In Eq. (1), $a$ and $b$ define the position and slope of the central (linear) region of the profile. In Eq. (2), $B_{1}$ and $B_{2}$ control the slope and width of the bell curves, respectively. In Eq. (3), $a_{1}, b_{1}, a_{2}, b_{2}, a_{3}$, and $b_{3}$ are the positions and slopes of the three single linear profiles. Tables 1-3 detail all the profiles that we prescribe.

Table 1: Shape parameters of prescribed linear profiles.

\begin{tabular}{lcc}
\hline Profile Name & $\boldsymbol{a}$ & $\boldsymbol{b}$ \\
\hline $\mathrm{L}-1$ & 1.5 & -2 \\
$\mathrm{~L}-2$ & 2.5 & -4 \\
\hline
\end{tabular}

Table 2: Shape parameters of prescribed bell-shape profiles.

\begin{tabular}{lcc}
\hline Profile Name & $\boldsymbol{B}_{\boldsymbol{1}}$ & $\boldsymbol{B}_{\boldsymbol{2}}$ \\
\hline BS-1 & 6 & 0.2 \\
BS-2 & 10 & 0.2 \\
BS-3 & 10 & 0.3 \\
\hline
\end{tabular}

Table 3: Shape parameters of prescribed piecewise linear profiles.

\begin{tabular}{lcccccc}
$\begin{array}{l}\text { Profile } \\
\text { Name }\end{array}$ & $\boldsymbol{a}_{\boldsymbol{1}}$ & $b_{\boldsymbol{1}}$ & $\boldsymbol{a}_{\mathbf{2}}$ & $\boldsymbol{b}_{\mathbf{2}}$ & $\boldsymbol{a}_{\mathbf{3}}$ & $\boldsymbol{b}_{\mathbf{3}}$ \\
\hline PL-1 & 0.5 & -1.8 & 1.1 & -1.8 & 1.7 & -1.8 \\
PL-2 & 1.3 & -4.8 & 1.1 & -1.8 & 0.568 & -0.6 \\
\hline
\end{tabular}

We use the Y-mixer to generate simple linear profiles and the cross-mixer for bell-shape profiles. In both cases, we fix channel dimensions and solution concentrations while varying the flow rates in each inlet channel during the optimal design process. To produce piecewise linear profiles, we join three Y-mixers. Here, we fix channel dimensions and subscribe the same flow rate for all inlet channels while adjusting the solution concentration in each inlet during optimization. The channel geometries and dimensions of each device as well as either the normalized solution concentration or flow rate in each inlet are shown in Fig. 2.

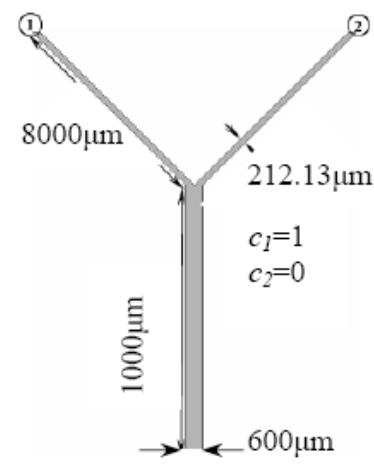

(a)

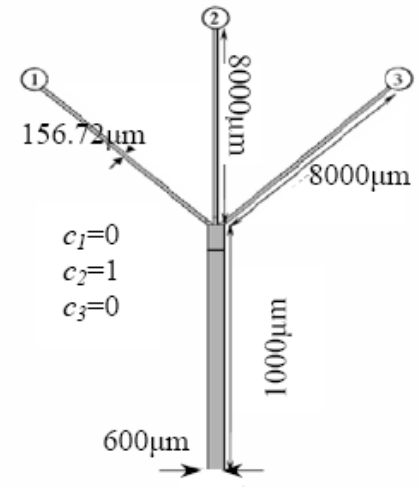

(b)

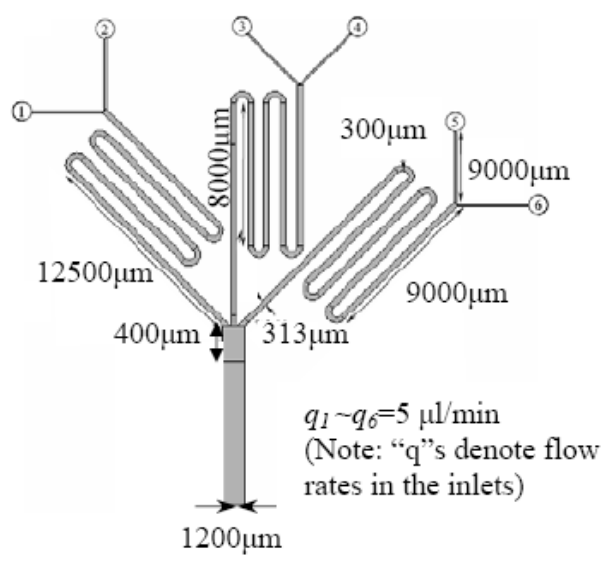

(c)

Fig. 2: Specified channel geometries and input concentration or flow rates: (a) Y-mixer, (b) cross-mixer and (c) triple $Y$-mixer.

\section{EXPERIMENTAL VALIDATION Fabrication}

Next, we fabricate microchips (e.g. Fig. 3) based on the designs. The $60 \mu \mathrm{m}$-deep micro-channels are fabricated in polydimethylsiloxane (PDMS) using standard soft lithography and rapid prototyping. The feature side of the PDMS is then treated in ultra-violet (UV) ozone for $5 \mathrm{~min}$ and bonded to a piece of glass slide which is cleaned and treated in $\mathrm{O}_{2}$ plasma for $15 \mathrm{sec}$. Finally, microfluidic connections are made at the chip inlets and outlets.

\section{Microfluidic Experiments}

Fluorophore Alexa 488 solutions (in phosphate buffer saline, or PBS) of different concentrations and PBS are 


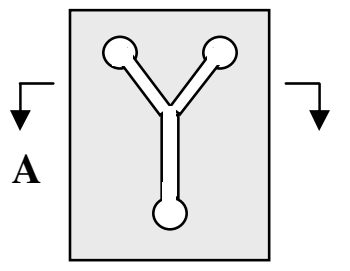

(a)

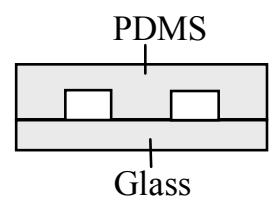

A-A
Fig. 3: Device fabrication: (a) top view and (b) cross-sectional view of the cross section $A-A$.

driven into the micro-channels by syringe pump (KD230P, KD Scientific). The experimental setup is shown in Fig. 4.For Y-mixer and cross-mixer, fluorescent images are taken at $10 \mathrm{~mm}$ downstream of the channel junction, while for triple-Y mixer, images are taken at $400 \mu \mathrm{m}$ downstream of the junction in the main channel. Intensity profiles across channel width are extracted from the fluorescent images.

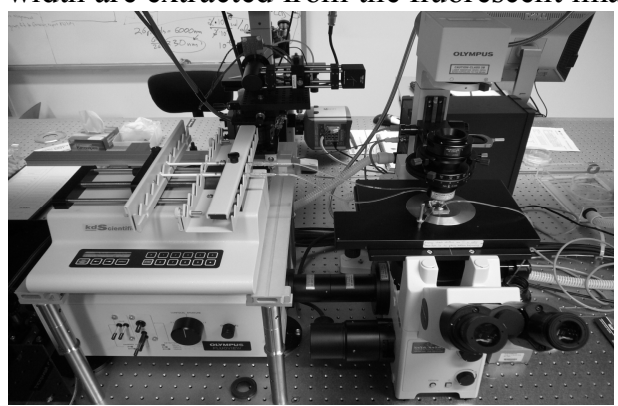

Fig. 4: Microfluidic experiment setup.

\section{RESULTS AND DISCUSSION}

With prescribed concentration gradient profiles and fixed channel dimensions, we specify inlet concentrations and use the synthesis method to obtain optimized inlet flow rates for the linear and bell-shape profiles, and we specify inlet flow rates and obtain optimized inlet concentrations for the piecewise linear profiles. The optimal flow rates for Yand cross-mixers are listed in Tables 4 and 5, and the optimal inlet concentrations for triple Y-mixer are listed in Table 6.

To verify the synthesis methodology as well as the analytical gradient generator model, we first compute the concentration gradient profiles from the synthesis results. We then conduct experiments, and the measured concentration profiles were compared with those from the synthesis (Figs. 5-7). The abscissas in the figures are the normalized lateral position, i.e. the distance between the data point and the left channel wall normalized by the channel width. The ordinates are normalized concentration, i.e. the sample concentration normalized by a reference concentration $(10 \mu \mathrm{M}$ in these cases). To facilitate the comparison of synthesis and experimental results, we define the follow parameter:

$$
E=\sum_{i=1}^{n}\left|c(i)-c_{D}(i)\right| / \sum_{i=1}^{n} c_{D}(i)
$$

where $c(i)$ and $c_{D}(i)$ are respectively the concentrations of the $i$ th data point on the experimental and designed profile curves, and $n$ is the number of data points. Note that this parameter captures the discrepancy between the theoretical and experimental results. From the figures, it can be seen that the experimental data agree well with the designed profiles, with maximum discrepancies $E=4.2 \%, 3.7 \%$ and $9.4 \%$ for the linear, bell-shape and piecewise linear profiles, respectively.

Table 4: Design synthesis results for linear profiles.

\begin{tabular}{lcc}
\hline Profile Name & $\begin{array}{c}\text { Sample Flow Rate } \\
(\mu \mathbf{l} / \mathbf{m i n})\end{array}$ & $\begin{array}{c}\text { Buffer Flow Rate } \\
(\mu \mathbf{l} / \mathbf{m i n})\end{array}$ \\
\hline L-1 & 0.722 & 0.722 \\
L-2 & 3.445 & 3.445 \\
\hline
\end{tabular}

Table 5: Design synthesis results for bell-shape profiles.

\begin{tabular}{lcc}
\hline Profile Name & $\begin{array}{c}\text { Sample Flow Rate } \\
(\mu \mathbf{l} / \mathbf{m i n})\end{array}$ & $\begin{array}{c}\text { Buffer Flow Rate } \\
(\mu \mathbf{l} / \mathbf{m i n})\end{array}$ \\
\hline BS-1 & 2.532 & 0.844 \\
BS-2 & 6.594 & 2.198 \\
BS-3 & 3.295 & 4.395 \\
\hline
\end{tabular}

Table 6: Design synthesis results for piecewise linear profiles.

\begin{tabular}{lcccccc}
\hline Profile & \multicolumn{7}{c}{ Inlet Normalized Concentration } \\
Name & $\mathbf{1}$ & $\mathbf{2}$ & $\mathbf{3}$ & $\mathbf{4}$ & $\mathbf{5}$ & $\mathbf{6}$ \\
\hline PL-1 & 0.408 & 0 & 0.408 & 0 & 0.408 & 0 \\
PL-2 & 1 & 0 & 0.408 & 0 & 0.136 & 0 \\
\hline
\end{tabular}

While this comparison of design and experimental concentration profiles establishes the validity of our synthesis method, it is interesting to examine possible cause for the discrepancies observed. These discrepancies are attributable to both the model and experiments. On the modeling side, a large channel width-to-depth ratio is assumed [8], so the depthwise mass transfer is neglected. Furthermore, a uniform cross-sectional velocity profile is assumed based on the large-aspect-ratio assumption. This causes discrepancies in the profiles near the channel walls. On the experimental side, the fabrication inaccuracy in channel dimensions and cross-sectional geometries is a source of the profile discrepancies. Also, as it is very difficult to superimpose the experimental profiles over the designed ones perfectly, a small offset of the profiles contributes to the discrepancies as well, especially at locations where steep slopes are present in the profile. This also explains why discrepancies are generally larger for piecewise linear profiles, which have two very steep transitions in the concentration profiles.

We now further analyze the trend exhibited by the concentration profiles obtained from the design and experiments. For the linear profiles (Fig. 5), we observe steeper concentration gradients at higher flow rates. This is because at higher flow rates, the sample residence time, $t$, is small, and hence the transverse diffusion length, $L=(2 D t)^{1 / 2}$, is small ( $D$ is the sample's diffusivity), which results in steeper concentration gradients. However, the linearity only exists in the central region of the channel, and the width of the linear region tends to shrink at higher flow rates. These are determined by the inherent physics of convectiondiffusion systems. Similarly, for bell-shape profiles (Fig. 6), the slopes of the bell curves are modulated by the inlet flow rates as well. In addition, the widths of the bell curves can be 
controlled by varying the ratio of flow rates between the middle and side channels of the cross-mixer. Higher middle-to-side-channel flow rate ratio leads to wider bell curve. In the case of piecewise linear profiles (Fig. 7), the three constitutive linear profiles are successfully realized except for slight roundups at the transition regions. To ensure sharp transitions between the linear profiles, observation point is close to the channel junction $(400 \mu \mathrm{m})$ rather than far down stream $(10 \mathrm{~mm})$ as in the other two cases.
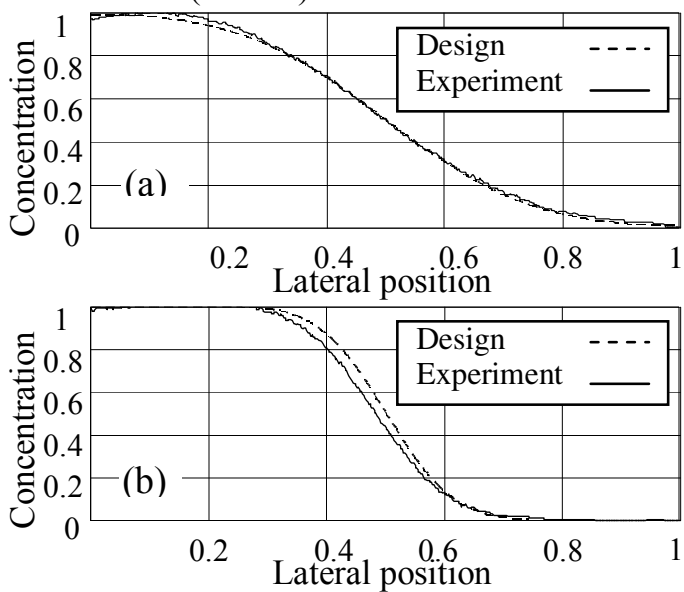

Fig. 5: Designed and experimental linear profiles: (a) Profile $L-1(E=2.8 \%)$ and (b) Profile $L-2(E=4.2 \%)$.
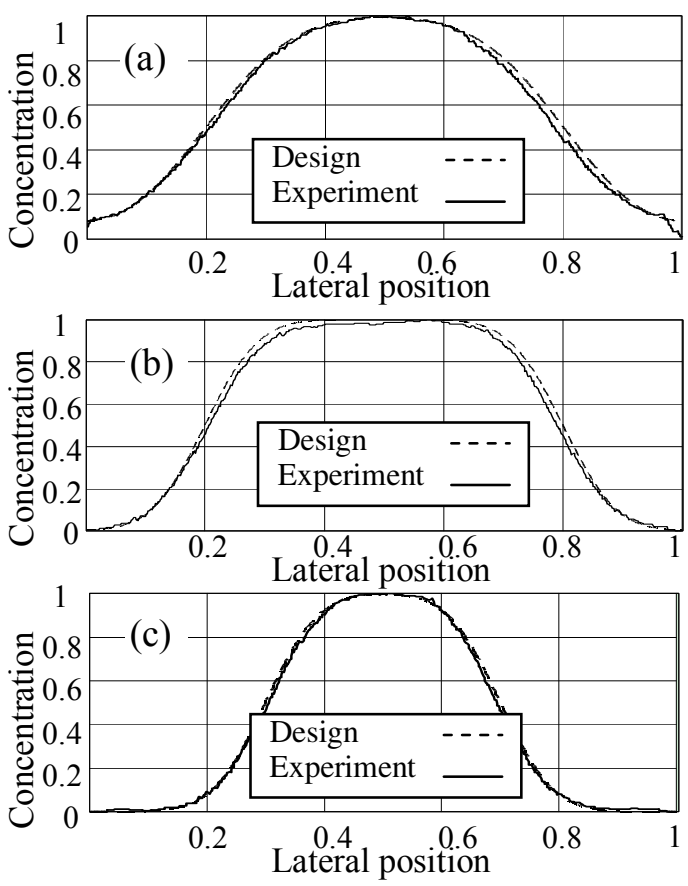

Fig. 6: Designed and experimental bell-shape profiles:(a) Profile $B-1(E=3.0 \%)$, (b) Profile B-2 $(E=3.7 \%)$ and (c) Profile B-3 $(E=3.0 \%)$.

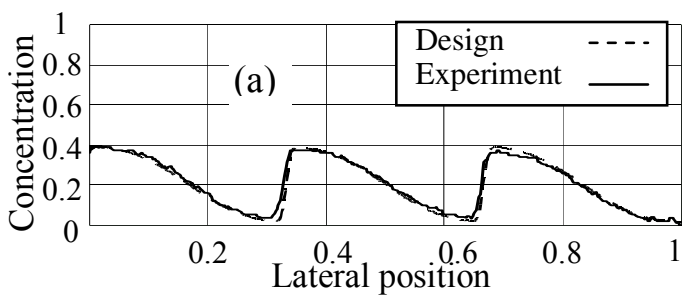

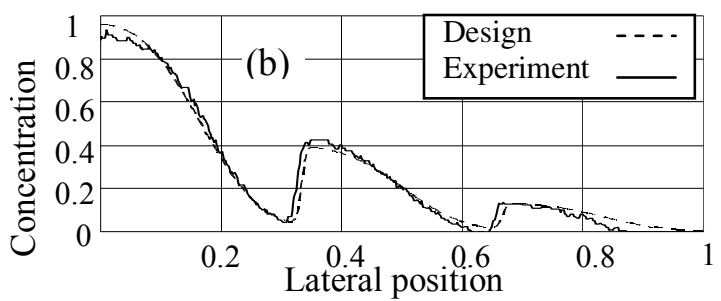

Fig. 7: Designed and experimental piecewise linear profiles: (a) Profile PL-1 $(E=7.3 \%)$ and (b) Profile PL-2 $(E=9.4 \%)$.

\section{CONCLUSIONS}

A synthesis method is presented to enable the efficient design of microfluidic concentration gradient generators for both simple and complex concentration profiles. The method is validated by comparing the designed and experimentally observed concentration profiles. Based on an analytical convection-diffusion model, the method leads to much improved computational efficiency comparing to numerical simulations. This validated synthesis method allows fast and optimal design and virtual prototyping of devices tailor-designed for specific cell biology experiments.

\section{ACKNOWLEDGEMENTS}

This work was sponsored by DARPA and the Air Force Research Laboratory, Air Force Material Command, USAF (grant no. F30602-01-2-0587), and the NSF ITR program (award no. CCR-0325344).

\section{REFERENCES}

[1] G. M. Walker, M. S. Ozers, and D. J. Beebe, "Cell Infection within a Microfluidic Device Using Virus Gradients", Sen. Act. B, vol. 98, pp. 347-355, 2004.

[2] N. L. Jeon et al., "Neutrophil Chemotaxis in Linear and Complex Gradients of Interleukin-8 Formed in A Micofabricated Device", Nat. Biotech., vol. 20, pp. 826-830, 2002.

[3] S. J. Wang et al., "Diffenrent Effects of EGF Gradient Profiles on MDA-MB-231 Breast Cancer Cell Chemotaxis", Exp. Cell Res., vol. 300, pp. 180-189, 2004.

[4] S. K. W. Dertinger et al., "Generation of Gradients Having Complex Shapes Using Microfluidic Networks", Anal. Chem., vol. 73, pp. 1240-1246, 2001.

[5] N. L. Jeon et al., "Generation of Solution and Surface Gradients Using Microfluidic Systems", Langmuir, vol. 16, pp. 8311-8316, 2000.

[6] X. Y. Jiang et al., "A General Method For Patterning Gradients of Biomolecules on Surfaces Using Microfluidic Networks", Anal. Chem., vol. 77, pp. 2338-2347, 2005.

[7] M. A. Holden et al., "Generating Fixed Concentration Arrays in A Microfluidic Device", Sen. Act. B, vol. 92, pp. 199-207, 2003.

[8] Y. Wang, T. Mukherjee, and Q. Lin, "Systematic Modeling of Microfluidic Concentration Gradient Generators", J. Micromech. Microeng., vol. 16, pp. 2128-2137, 2006. 